\title{
Descripción imagenológica de un caballo criollo colombiano con enfermedad articular degenerativa del tarso
}

\author{
Imaging description of a colombian creole horse with degenerative \\ joint disease of the tarsus
}

Gallego-Rodriguez RS, Álvarez-Mejía CP, López-Vera LA. Descripción imagenológica de un caballo criollo colombiano con enfermedad articular degenerativa del tarso. Rev Colombiana Cienc Anim. Recia. 2020; 12(1):e736. https://doi.org/10.24188/recia.v12.n1.2020.736

Universidad de Sucre, Colombia

Los autores permiten a RECIA reimprimir el material publicado en él. En caso de que un autor quiera traducir o usar una publicación parcial o completa de nuestro Diario, el autor debe obtener un permiso por escrito del editor de la revista.

Copyright (C) 2020. El (los) autor (es), Revista Colombiana de Ciencia Animal - RECIA. 2020. Este es un artículo de acceso abierto distribuido bajo los términos de Creative Commons Attribution 4.0 (https://creativecommons.org/licenses/by-nc-sa/4.0/), El uso, distribución o reproducción está permitido, siempre que se acrediten al autor original y al propietario del copyright y que se cite la publicación original en esta revista, de acuerdo con la práctica académica aceptada. No se permite el uso, distribución o reproducción que no cumpla con estos términos.

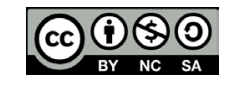




\title{
Descripción imagenológica de un caballo criollo colombiano con enfermedad articular degenerativa del tarso
}

\author{
Imaging description of a colombian creole horse with degenerative joint disease of the \\ tarsus
}

Renso Sneider Gallego-Rodriguez. M.Sc.

DOI: https://doi.org/10.24188/recia.v12.n1.2020.736

Corporación Universitaria Remington, Facultad de Medicina

Veterinaria, Grupo de Investigación en Veterinaria (GINVER),

Medellín, Colombia.

renso.gallego@uam.edu.co

(i) https://orcid.org/0000-0003-1563-9731

Claudia Patricia Álvarez-Mejía. MV.

Corporación Universitaria Remington, Facultad de Medicina Veterinaria, Medellín, Colombia.

pat_mdb@hotmail.com

(i) https://orcid.org/0000-0002-3249-8048

Luis Alejandro López-Vera. MV.

Corporación Universitaria Remington, Facultad de Medicina

Veterinaria, Medellin, Colombia.

luisalejo0611@uniremington.edu.co

(iD) https://orcid.org/0000-0003-2780-6234

Recepción: 15 noviembre 2019

Aprobación: 8 junio 2020

Publicación: 12 junio 2020

\section{RESUMEN}

En este artículo se reporta un caso clínico de una yegua criolla de 4 años, que llega a consulta por cojera y aumento de tamaño de la articulación del tarso en el miembro posterior derecho producto de un traumatismo. Se realiza examen clínico de la paciente en el cual se evidencia una claudicación de apoyo 5/5 con notables movimientos compensatorios; a la palpación refiere dolor y se evidencia tumefacción, aumento de temperatura y del tamaño. Se realizó hemograma, química sanguínea, evaluación del líquido articular por medio de citología y cultivo; a su vez, se realiza diagnóstico imagenológico mediante radiografía convencional y ecografía articular, el mismo se hace durante varios períodos de la evolución del caso, se realiza radiografía convencional en la cual se encuentran hallazgos como reacción proliferativa, presencia de osteofitos, anquilosis, entre otros; la evaluación ecográfica presentó hallazgos como disminución del líquido sinovial, aumento de tamaño y de ecogenicidad de la membrana sinovial y presencia de bandas de fibrina presentes en el líquido articular. El abordaje terapéutico se realiza mediante fármacos analgésico, lavado articular e infiltración con corticoides, e infusión intraarticular con DMSO al 10\%. El presente caso describe el seguimiento imagenológico mediante ecografía y radiografía, hecho en un paciente equino con enfermedad articular degenerativa del tarso, lo cual entrega herramientas útiles a la comunidad veterinaria en el conocimiento y la interpretación de los cambios radiográficos y ultrasonográficos en un equino con enfermedad articular degenerativa durante el tiempo de evolución patológica.

Palabras claves: Artritis, diagnóstico, ecografía, equinos, imagenología, radiología. 


\begin{abstract}
This article reports a clinical case of a 4-year-old Creole mare, who comes to consultation due to lameness and an increase in the size of the tarsal joint in the right hind limb resulting from trauma. A clinical examination of the patient is performed in which a 5/5 support claudication is evident with notable compensatory movements; to palpation refers pain and swelling is evident, increase in temperature and size. Hemogram, blood chemistry, evaluation of joint fluid was performed through cytology and culture; in turn, an imaging diagnosis is made during several periods of the evolution of the case, conventional radiography is performed in which there are findings such as proliferative reaction, presence of osteophytes, ankylosis, among others; The ultrasound evaluation presented findings such as decrease in synovial fluid, increase in size and echogenicity of the synovial membrane and presence of fibrin bands present in the joint fluid. The therapeutic approach is performed through analgesic drugs, joint lavage and corticosteroid infiltration, and intraarticular infusion with $10 \%$ DMSO. The present case describes the imaging follow-up of an equine patient with degenerative joint disease of the tarsus, which provides tools to the veterinary community for the correct diagnosis of joint disease in the equine.
\end{abstract}

Keywords: Arthritis; diagnosis; ultrasound; equines; imaging, radiology.

\title{
INTRODUCCION
}

Las articulaciones juegan un papel muy importante en el desplazamiento del animal manteniendo una estructura e integridad adecuada de sus extremidades y proporcionando un soporte adecuado (1). La enfermedad articular es la causa más común de cojeras en equinos, se relaciona con factores como traumatismo constante, inestabilidad articular, infecciones y alteraciones de la osificación endocondral (2).

Las articulaciones con más afección son las articulaciones del carpo, del tarso e interfalángicas, las cuales soportan mayor peso y presentan mayor movilidad en el equino (3). Son diversos los factores proinflamatorios que afectan la articulación, considerando al factor de necrosis tumoral (TNF $\alpha$ ) y la interleucina 1 (IL-1) como los dos principales mediadores de la respuesta inflamatoria, también participan otras interleucinas, factores de necrosis, entre otros. Se ha descrito que las reacciones enzimáticas que se presentan en la enfermedad articular son mediadas por la vía clásica del sistema del complemento (4).

Los métodos diagnósticos relacionados con la enfermedad articular abarcan desde el examen físico, goniometría articular, evaluación del líquido sinovial, técnicas imagenológicas, entre otros; el análisis del líquido sinovial se aborda desde el aspecto físico donde se evalúa la viscosidad, la turbidez, y el color, el aspecto celular, donde se tiene en cuenta la reacción leucocitaria y las proteínas plasmáticas y el aspecto microbiológico, en el cual mediante la técnica de cultivo se evidencia el crecimiento bacteriológico (5).

El tarso en el equino es una de las regiones más afectadas y uno de los principales aspectos anatómicos que dan origen a ciertas claudicaciones, siendo el diagnóstico por imagen común e indispensable en el abordaje clínico (6). La evaluación de las anormalidades en la región tarsal se realiza de manera complementaria, teniendo en cuenta el uso de técnicas convencionales como la radiografía y la ecografía articular, donde de manera complementaria pueden ser visualizadas estructuras densas como el tejido óseo y aspectos relacionados al tejido blando articular (7). El uso de técnicas imagenológicas más especializadas como lo son la tomografía computarizada, la resonancia magnética y la artroscopia permiten la visualización y la ubicación directa de los hallazgos articulares generando un diagnóstico con mayor sensibilidad (8).

El estudio radiográfico relacionado a los hallazgos del examen físico, es la ayuda diagnóstica más utilizada y certera para el reconocimiento de esta patología de mayor prevalencia en equinos. La formación de osteofitos, disminución del espacio articular y la esclerosis del hueso subcondral son los hallazgos radiológicos más comunes observados en las placas. Debido a su componente inflamatoria (Sinovitis) puede estar acompañado de dolor, pérdida de función, tumefacción, aumento de tamaño y de temperatura (2). La mayor desventaja de los rayos x es que en etapas tempranas de la enfermedad articular no se observa el deterioro del cartílago articular y esto atrasa el diagnóstico y tratamiento lo que va a empeorar el pronóstico (3). 
El ultrasonido permite la posibilidad de observar anormalidades sobre los tejidos blandos como la vaina sinovial, cápsula articular y ligamentos relacionados a la articulación. Esta técnica se utiliza en casos en los que se reporta distención articular por efusión de líquido sinovial, inflamación local y dolor en la manipulación o intentos de realizar los movimientos normales de la articulación. Mediante esta técnica se pueden observar también cambios en la superficie ósea que siempre deben ir relacionados a los hallazgos del estudio radiográfico (3). El presente caso clínico pretende dar a conocer los cambios radiográficos y ultrasonográficos del paciente equino con enfermedad articular a lo largo de su evolución patológica.

\section{DESCRIPCION DEL CASO}

Anamnesis. Equino hembra, raza criolla de pelaje pinto colorado, con edad de 4 años, $325 \mathrm{~kg}$ de peso vivo se presenta a consulta a la Clínica Veterinaria de la Corporación Universitaria Remington, con historial de un trauma ocurrido 5 meses atrás en la región del tarso del miembro posterior derecho.

Examen Físico. Se realiza evaluación clínica al momento de llegada del paciente, el cual presenta, claudicación unilateral $5 / 5$, hipotrofia de los músculos glúteos derechos, dolor al realizar contacto con el tarso derecho, aumento de tamaño y aumento de temperatura de la región afectada. En la inspección se evidencia cicatriz en el aspecto lateral de la articulación tarsocrural del miembro posterior derecho compatible con el sitio del trauma, la zona se palpa tumefacta y dura con algunas zonas pequeñas de efusión sinovial, se hace medición del perímetro articular desde la región dorsal del tarso hacia la tuberosidad calcanea, obteniendo un resultado de $39 \mathrm{~cm}$.

Se realizan pruebas dinámicas con el fin de evaluar el o los sitios de afección presentes en el miembro posterior de la paciente, al desplazamiento se clasifica como claudicación de apoyo con un grado 5/5, son evidentes movimientos compensatorios debido al dolor, apoyando en pinza mientras está en estación, se realizan bloqueos perineurales con el fin de evaluar la sensibilidad del miembro y permitir analgesia para realizar artrocentesis, al realizar los bloqueos tibial y peroneo el grado de claudicación disminuye a 3/5 y hay apoyo del aspecto solar del casco en estación.

Examen Imagenológico. Se decide hacer una ecografía articular inicial con el fin de evaluar los tejidos blandos, el líquido articular y realizar la técnica de artrocentesis ecoguiada (Figura 1), el ultrasonido articular se realiza con un ecógrafo Emperor medical G30 con una sonda lineal a una frecuencia de $9 \mathrm{MHz}$, mediante el abordaje lateral de la articulación tarsocrural se observa distensión de la membrana sinovial, con aumento del volumen del líquido sinovial y cambios significativos en su ecogenicidad, ya que se observa hipoecogénico, de ecotextura heterogénea, con presencia de material vegetativo relacionado a bandas de fibrina, las cuales podrían indicar hemorragia intraarticular o hemartrosis, a su vez, se evidencia irregularidad del cartílago articular, sugiriendo un proceso de erosión del mismo.

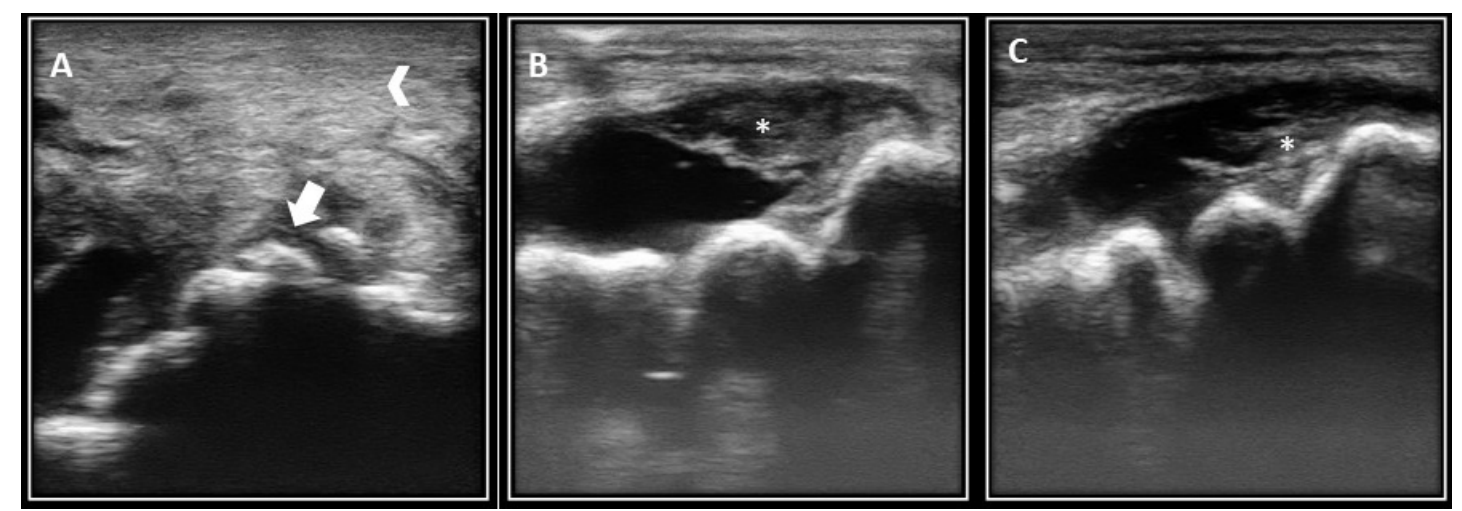

Figura 1. A. Inicio del caso, se observa aumento del tamaño y de la ecogenicidad de la membrana sinovial con presencia escasa de líquido sinovial (punta de flecha), a su vez, se identifica la presencia de estructuras de radiopacidad mineral compatibles con reacción perióstica. B. (tiempo de evolución de 15 días). C. Tiempo de evolución 4 meses, se evidencia presencia de bandas compatibles con fibrina a nivel del líquido sinovial.

Al realizar el abordaje ecográfico en la porción plantar y medial de la articulación se encuentra aumento severo de tamaño del tejido blando articular con aumento de su ecogenicidad ya que se observó hipoecogénico heterogéneo con mínima cantidad de líquido sinovial, a lo largo de la evolución del caso los hallazgos ecográficos mostraron una disminución marcada del líquido sinovial, con presencia de múltiples estructuras hiperecogénicas de bordes irregulares compatibles con osteofitos, así mismo, el tejido blando aumentó de tamaño y de ecogenicidad. 
Según lo observado en el ultrasonido se decide realizar la punción articular en la porción lateral de la articulación tarsotibial; el examen macroscópico del líquido articular muestra un aspecto turbio, sanguinolento con disminución moderada de la viscosidad. Se decide enviar a laboratorio dos muestras del líquido sinovial para la evaluación citológica y cultivo bacteriano y una muestra de sangre para química sanguínea (BUN, creatinina, AST) y hemograma.

Según lo encontrado al examen clínico y la ecografía se decide realizar un estudio radiológico ortogonal de tarso y de menudillo (desplazamiento de la articulación interfalángica proximal en el examen clínico) del miembro posterior derecho, dicho estudio se realizó varias veces a lo largo de la evolución del caso clínico, siendo que se tomaron vistas radiográficas en el momento inicial del caso, a los 15 días posteriores, a los 4 meses después y un año después (Figura 2). En el examen inicial se realiza una vista lateral del tarso se observa un área de proliferación y reacción perióstica moderada en el aspecto dorsal de la porción distal de la tibia, así mismo en la porción dorsal del tarso central y del tercer hueso tarsiano, la fosa lateral del talus presenta un aspecto radiopaco poco definido, no es posible observar el espacio de la articulación talo-calcanea lateral, evidencia los tubérculos del calcáneo de aspecto poroso, se observa aumento del tamaño del tejido blando en el aspecto dorsal de la articulación (Figura 3).
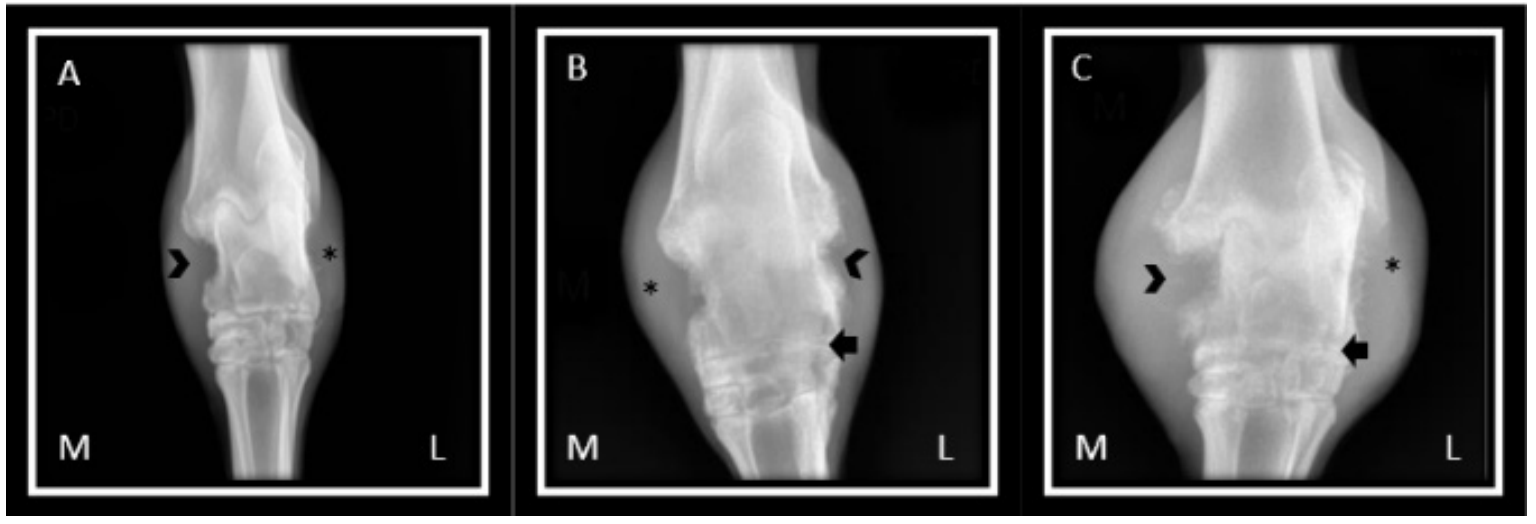

Figura 2. Radiografía anteroposterior del tarso, se observa marcada reacción perióstica - proliferativa, relacionada a pérdida de la densidad ósea en la porción media y lateral del tarso (punta de flecha), así mismo, anquilosis en la articulación intertarsiana proximal (flecha), se evidencia severo aumento de tamaño del tejido blando periarticular (*). La radiografía A corresponde al momento inicial del caso, radiografía B, fue hecha 15 días después, radiografía $C$ fue realizada 4 meses después.
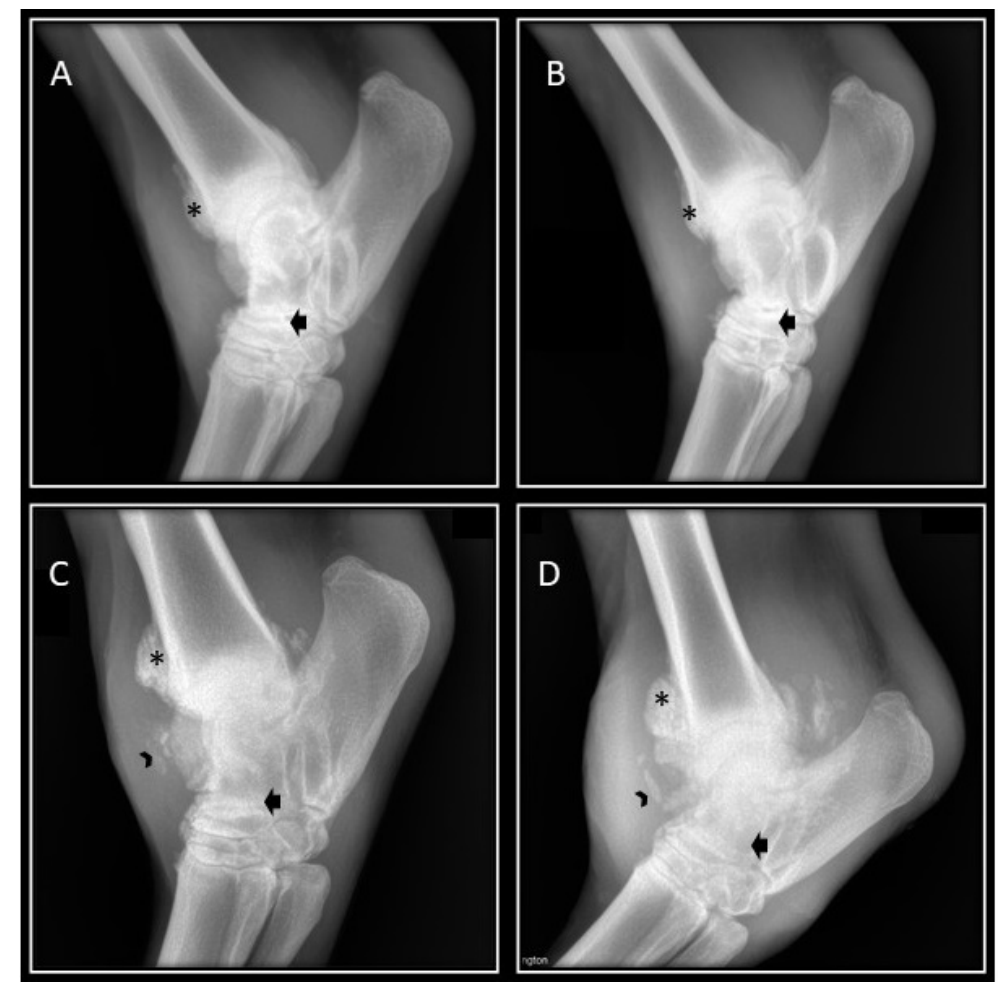

Figura 3. Radiografía lateral de tarso en la cual se observa reacción proliferativa en la región distal de la tibia (*), se observa un proceso de lisis ósea generalizada en el aspecto dorsal del astrágalo (punta de flecha), a su vez, se evidencia pérdida de los espacios articulares (flecha), reacción osteofítica marcada y aumento de la densidad del tejido blando periarticular. La radiografía A, corresponde al momento inicial del caso, la radiografía B, se realizó 15 días después, radiografía C, se realizaron 4 meses después, radiografía $\mathrm{D}$, un año después. 
En el abordaje anteroposterior se encuentra proliferación ósea y reacción perióstica del maléolo medial de la tibia el cual presenta una superficie irregular y disminución de la densidad, lo cual, se hace generalizado para el aspecto medial y lateral del astrágalo, se observa disminución del espacio articular entre las articulaciones intertarsianas proximales e intertarsianas distales, hay lisis óseas en el cuarto hueso tarsiano, finalmente se hace evidente el aumento de tamaño del tejido blando en los aspectos mediales y laterales de la articulación tarsiana . En la radiografía lateral del menudillo se encuentra luxación de la articulación interfalángica proximal, los sesamoideos proximales se observa con un patrón apolillado con múltiples zonas focales de lisis ósea y perdida de densidad. Con la evolución del proceso inflamatorio articular se evidenciaron severos cambios relacionados con lisis ósea, aumento de la reacción proliferativa, y deformación severa de la articulación tarsotibial, estos procesos fueron observados a los 4 meses y al año posterior al inicio del caso clínico.

Pruebas paraclínicas. Los resultados del hemograma mostraron una leve tendencia a la anemia, leve tendencia a leucocitosis, con linfocitosis leve e hiperproteinemia. No se reportó crecimiento bacteriano a la muestra enviada para cultivo articular, en la citología se reportó que la consistencia sinovial era líquida, de color rojo, con aspecto turbio; a nivel microscópico se observa moderada reacción leucocitaria con predominio de neutrófilos (68\%), mononucleares (31\%), eosinófilos esporádicos (1\%), compatible con artropatía inflamatoria.

Según el plan diagnóstico realizado y los hallazgos al examen clínico se cuenta como diagnóstico presuntivo osteoartritis del tarso en el miembro posterior derecho, acompañado de luxación de la articulación interfalángica proximal. Posterior a los cuatro meses de evolución patológica de la paciente se decide realizar artrotomía con el fin de evaluar el tejido periarticular y extraer una muestra para histopatología, de los cuales el resultado concluyó que los fragmentos evaluados estaban constituidos por abundante tejido conectivo denso regular con fibroblastos típicos y neovascularización, también se encontró tejido con abundante colágeno denso regular e islotes óseos con trabéculas típicas y abundantes osteocitos, lo cual fue compatible con un diagnóstico de enfermedad articular degenerativa.

Terapéutica. A nivel terapéutico se instauró un tratamiento dirigido hacia el manejo del dolor, de manera sistémica se administran $2.2 \mathrm{mg} / \mathrm{kg}$ de ketoprofeno durante 5 días, acompañados de una terapia antiulcerogénica con sucralfato 30 $\mathrm{mg} / \mathrm{kg}$ vía oral cada 12 horas durante 10 días; con el fin de generar un efecto higroscópico sobre las zonas de efusión sinovial se hacen paños con sulfato de magnesio a una dosis de 100 gr/2 litros, vía tópica cada 12 horas por 15 días; se realizó infiltración mediante la técnica ecoguiada de las articulaciones tarsocrural y tibiotarsiana con Kenacort ${ }^{\mathrm{R}}$ (acetato de triamcinolona) a 9mg/articulación, se recomendó reposo absoluto de la paciente con descanso en cama profunda; como suplemento articular se suministra $\operatorname{Artiflex}^{\mathrm{R}}$ (glucosamina, aceite de soya, vitamina A, vitamina E, condroitina, ácidos grasos esenciales) a una dosis de $25 \mathrm{ml}$ /vía oral cada 24 horas durante 30 días.

Una semana después se hace medición de la articulación con un valor de $34 \mathrm{~cm}$, el cual continúa sobre las semanas posteriores, a la tercera semana se evidencia un aumento de tamaño articular con un valor del perímetro de $39 \mathrm{~cm}$, para ese momento se decide realizar un lavado articular con 50 gramos de DMSO (dimetilsulfóxido) diluidos en 500 ml de solución salina fisiológica, el líquido articular que se recupera del lavado es de aspecto turbio y color rojo, posterior al lavado se infiltra Kenacort ${ }^{\mathrm{R}} 25 \mathrm{mg}$ totales en la articulación tarsotibial y $10 \mathrm{mg}$ totales en la articulación intertarsiana proximal, al día siguiente se observa disminución de la claudicación de 4/5 a 2/5.

\section{DISCUSION}

Las lesiones articulares de tarso en equinos son comunes, ya sean de origen primario óseo o producidas por un evento secundario (9). Osteoarthritis (OA; a criterio de los autores, la relación entre los hallazgos al examen clínico y las técnicas imagenológicas permiten evidenciar las estructuras afectadas (tejido blando, tejido óseo, cavidad articular), el tipo de daño, y probablemente la cronicidad de las lesiones.

En caballos adultos se ha descrito que la osteoartritis puede resultar secundaria a un evento infeccioso - inflamatorio a nivel intraarticular, lo anterior se puede relacionar a terapias inadecuadas, utilización incorrecta de algunos fármacos, mala praxis, entre otros (10). Promising reports on the clinical use of poly(methyl methacrylate. Según lo reportado en la anamnesis del paciente la causa inicial fue un traumatismo en el aspecto lateral de la articulación tarso-crural, el cual produjo efusión articular, aumento de la temperatura de la zona y claudicación; fueron realizados varios tratamientos sin respuesta positiva, derivando en una mayor reacción inflamatoria articular.

Hardy (11), menciona que el estudio radiográfico en pacientes con enfermedad articular es necesario ya que puede correlacionarse con los hallazgos clínicos presentes en el examen físico del paciente, a su vez describe lesiones como disminución de la opacidad a nivel del hueso cortical, reacción perióstica exacerbada, aumento del tamaño y la densidad 
de la cápsula articular y aumento del líquido sinovial (11), en un estudio realizado por Raes (12), encontró que el 69.7\% (62/89) de los animales evaluados tuvieron hallazgos radiográficos relacionados con lesiones compatibles con artritis (12), estos hallazgos radiológicos se relacionan con el estudio realizado ya que la paciente presentó aumento del tamaño articular, área de proliferación y reacción perióstica moderada en el aspecto dorsal de la porción distal de la tibia, porción dorsal del tarso central y del tercer hueso tarsiano; la fosa lateral del talus presenta un aspecto radiopaco poco definido, no es posible observar el espacio de la articulación talo-calcánea lateral, se evidencia los tubérculos del calcáneo de aspecto poroso, se observa aumento del tamaño del tejido blando en el aspecto dorsal de la articulación.

La ecografía puede indicar hallazgos relacionados con el tejido blando y la superficie perióstica a nivel articular (13). De acuerdo a lo anterior Caron (14), da prioridad a la ecografía con respecto a la radiografía convencional ya que permite la observación de anormalidades en las vainas sinoviales, cápsula articular, membrana sinovial y lesiones intraarticulares (14). Denoix (15), afirma que las indicaciones para la evaluación ecográfica articular incluyen distención por efusión sinovial, aumento de tamaño local, dolor a la manipulación pasiva y hallazgos significativos en la analgesia o anestesia periarticular (15). A pesar de que el ultrasonido se absorbe completamente en la porción cortical del hueso, las características periósticas pueden ser evaluadas, encontrando hallazgos como osteofitos periarticulares, entesófitos, fragmentos osteocondrales e irregularidades de la porción subcondral (14). Lo mencionado anteriormente se relaciona con la evaluación ecográfica del caso actual donde se encontraron hallazgos como aumento del tamaño y la ecogenicidad de la membrana sinovial, marcada disminución del líquido articular, irregularidad del borde perióstico, desprendimiento de fragmentos osteocondrales con presencia de sombra acústica. Durante el seguimiento ecográfico del caso se encontró un aumento progresivo del tejido inflamatorio, la ecogenicidad del mismo aumento drásticamente, a su vez, hubo una marcada disminución del líquido sinovial.

Autores como Hinz y Fischer (16), mencionan que la evaluación ecográfica de las articulaciones es más precisa en comparación a la radiografía con respecto a las lesiones del cartílago articular, según el estudio realizado se reportó que la precisión de la ecografía fue del 83\% en relación al 62\% de la radiografía, con una sensibilidad del 91\% comparado con el 67\% de la radiografía (16). Según la evaluación realizada en el presente caso se puede afirmar que las lesiones tempranas o agudas del cartílago articular pueden ser observadas únicamente mediante el examen ecográfico articular, a su vez, la radiografía se hace importante a medida que el cartílago se fractura, se separa y se mineraliza formando artrofitos radiopacos visibles mediante el estudio radiológico, lo cual indica que el estudio radiológico se hace importante en eventos patológicos crónicos.

La interpretación de técnicas imagenológicas avanzadas como parte del diagnóstico de enfermedad articular en el equino representa un reto clínico para el médico veterinario (17). Según autores como Blaik et al (18), en las evaluaciones realizadas mediante resonancia magnética encontraron que el $80 \%$ de las claudicaciones crónicas de baja intensidad se presentaban en el tarso, además, afirman que la resonancia magnética es la única técnica no invasiva que permite la evaluación directa del cartílago articular, el hueso subcondral, y los tejidos blandos periarticulares (18). Durante la evolución del presente caso se consideró dentro de los planes diagnósticos la realización de técnicas imagenológicas avanzadas como la tomografía computarizada o la resonancia magnética que permitieran una descripción más precisa de los hallazgos articulares, pero debido a consideraciones económicas no fue posible que se realizaran estas técnicas, a criterio de los autores se considera necesario el uso de ayudas como la resonancia magnética y la tomografía con el fin de obtener diagnósticos de mayor sensibilidad.

En conclusión, el examen radiológico y el ecográfico deben considerarse pruebas complementarias las cuales permitan identificar las lesiones articulares de manera más adecuada. En casos como la enfermedad articular degenerativa la radiografía permite obtener hallazgos como proliferación ósea, disminución de los espacios articulares, osteofitos, entre otros; por otro lado, la ecografía permite encontrar signos tempranos de lesión en el cartílago articular, irregularidad y erosión del mismo, de igual manera, es posible determinar la integridad de la membrana sinovial y los posibles cambios en su ecogenicidad.

\section{REFERENCIAS}

1. Rolando J, Polli M, Caggiano N, Perrone G. Aplicación de la determinación de las metaloproteinasas de la matriz (MMP) en las enfermedades articulares equinas. Fisiología animal y laboratorio del metabolismo óseo. 2013. https://www.researchgate.net/publication/291215128 
2. Weeren R. Joint physiology: responses to exercise and training. In: Equine Sports Medicine and surgery. Equine Sport Med Surg. 2014;213-222. https://doi.org/10.1016/B978-0-7020-4771-8.00011-9

3. Summer NH. Avances en Osteoartritis equina. 2012. Universidad Austral de Chile; Valdivia Chile

4. Polli M, Caggiano N, Perrone G, Marino M, De Simone E, Chiappe Barbará A. Variación del nivel de citoquinas en líquido sinovial de equinos con enfermedad articular tratados con biofosfonatos. Rev Complut Ciencias Vet. 2013; 7(1):69-83. https://doi.org/10.5209/rev RCCV.2013.v7.n1.42064

5. Steel C. Equine Synovial Fluid Analysis. Vet Clin Equine Pract. 2008; 24:437-454. https://doi.org/10.1016/j. cveq.2008.05.004

6. Vanderperren K, Raes E, Bree H Van, Saunders JH. Diagnostic imaging of the equine tarsal region using radiography and ultrasonography. Part 2: Bony disorders. Vet J. 2009;179(2):188-196. http://dx.doi.org/10.1016/j.tvjl.2007.08.025

7. Vanderperren K, Raes E, Hoegaerts M, Saunders JH. Diagnostic imaging of the equine tarsal region using radiography and ultrasonography. Part 1: The soft tissues. Vet J. 2009;179(2):179-187. http://dx.doi.org/10.1016/j. tvjl.2007.08.030

8. Mair TS, Kinns J, Jones RD, Bolas NM. Magnetic resonance imaging of the distal limb of the standing horse. Equine Vet Educ. 2005;17:74-78. http://www.bellequine.co.uk/downloads/mri of distal limbs eve 2005.pdf

9. Maria Verônica de Souza. Osteoarthritis in horses - Part 1: relationship between clinical and radiographic examination for the diagnosis. Brazilian Arch Biol Technol. 2016; 59:1-9. https://doi.org/10.1590/1678-4324-2016150024

10. Haerdi-Landerer MC, Habermacher J, Wenger B, Suter MM, Steiner A. Slow release antibiotics for treatment of septic arthritis in large animals. Vet J. 2010; 184(1):14-20. http://dx.doi.org/10.1016/j.tvjl.2009.02.013

11. Hardy J. Etiology, Diagnosis, and Treatment of Septic Arthritis, Osteitis, and Osteomyelitis in Foals. Clin Tech Equine Pract. 2006; 5(4):309-317. https://doi.org/10.1053/i.ctep.2006.09.005

12. Raes E V., Vanderperren K, Pille F, Saunders JH. Ultrasonographic findings in 100 horses with tarsal region disorders. Vet J. 2010; 186(2):201-209. https://doi.org/10.1016/j.tvjl.2009.07.026

13. Smith M, Smith R. Diagnostic ultrasound of the limb joints, muscle and bone in horses. In Pract. 2008; 30(3):152159. http://dx.doi.org/10.1136/inpract.30.3.152

14. Caron J. Arthritis: Osteoarthritis. In: Diagnosis and Management of Lameness in the Horse. Saunder Elsevie; 2011. https://www.elsevier.com/books/diagnosis-and-management-of-lameness-in-thehorse/9780721683423

15. Denoix J. Ultrasonographic Examination of Joints. In: Diagnosis and Management of Lameness in the Horse. In: Saunder Elsevier; 2011. http://www.equisan.com/images/pdf/ultrajoints.pdf

16. Hinz A, Fischer AT. Comparison of the Accuracy of Radiography and Ultrasonography for Detection of Articular Lesions in Horses. Vet Surg. 2011; 40(7):881-885. https://doi.org/10.1111/j.1532-950X.2011.00873.x

17. Fernandez J, Sanchez J, Alonso P, Orden MA. Magnetic resonance imaging in some equine limb injuries diagnosis. Different contributions of basic weighted sequences in the study and diagnosis of each injury. Redvet. 2010;(1):177190. https://www.redalyc.org/pdf/636/63613103015.pdf

18. Blaik, MA, Hanson RR, Kincaid SA, Hathocock JT, Hudson JA, Baird DK. Low-field magnetic resonance imaging of the equine tarsus: normal anatomy. Vet Radiol Ultrasound. 2000;41:131-141. https://www.vetmed.auburn.edu/ wp-content/uploads/2015/01/16-Low-field-magnetic-resonance-imaging-of-the-equine-tarsus-normal-anatomyVeterinary-Radiology-Ultrasound-2000.pdf 